\title{
Examining Atomistic Defect-Boundary Interactions Induced by Ion Irradiation using Aberration Corrected Transmission Electron Microscopy
}

\author{
J.A. Aguiar ${ }^{1}$, M. Chi ${ }^{2}$, P.G. Kotula ${ }^{3}$, Z. Bi ${ }^{1}$, O. Anderoglu ${ }^{1}$, K. Baldwin ${ }^{1}$, J.A. Valdez ${ }^{1}$, A. Misra ${ }^{1}$ and \\ B.P. Uberuaga ${ }^{1}$ \\ ${ }^{1}$ Material Science and Technology Division, Los Alamos National Laboratory, Los Alamos, NM, \\ 87545, USA \\ ${ }^{2}$ Division of Material Science and Technology, Oak Ridge National Laboratory, Oak Ridge, TN, 37831, \\ USA \\ ${ }^{3}$ Sandia National Laboratories, Albuquerque, NM, 87185-0886, USA
}

With current and future developments in field emission technology, the ability to resolve atomic structure and spectral features such as band gaps, optical transitions, and fine structural differences in materials using scanning transmission electron microscopy (STEM) and electron energy loss spectroscopy (EELS) is expanding at a increasing rate [1]. At the same time, recent developments within the nuclear materials community have lead researchers to hypothesize that composite materials can address concerns regarding nucleation, growth, and the migration of voids or inert gas bubbles and fission fragments at higher temperatures and radiation environments at the atomic scale [2, 3]. Advanced microscopy allows for unprecedented insight into the physical mechanisms responsible for the improved performance of these materials. For example, a recent study by Misra et al. [4] used high resolution TEM to show metallic multilayer composites with smaller layer thicknesses contained a larger number of defects at the interfaces that offered improved mechanical strength and resistance to both radiation induced swelling and embrittlement. To extend beyond these types of studies, and extract the potential changes in point defect chemistry and valence structure, the spatial and energetic limits associated with the current use of advanced aberration corrected and energy filtered microscopy is a necessary tool to address the underlying physical mechanisms responsible for material performance and behavior [5-10].

To address these issues, we have performed a series of quantitative comparisons between different processing methods, including principal component and multivariate statistical analysis, for performing analytical spectral imaging of irradiated oxide interfaces to address both the spectral expansion and resolution on a variety of transmission electron microscopes ranging from Schottky to cold field emission. Across all techniques considered [11-13], however, the need for tracking low lying spectral peaks in both the valence and core low loss limits has been previously reported [14], but till this point has not been applied to such distinct cases where radiation damage is used to probe the response of atomic scale composite heterointerfaces to swift energetic ions.

Applying these latest experimental and analytical capabilities, we have examined the evolution of interfacial electronic structure and chemistry at composite heterointerfaces, in particular $\mathrm{SrTiO}_{3} / \mathrm{CeO}_{2}$ and $\mathrm{Fe} / \mathrm{TiO}_{2}$, subjected to ion irraddiation using aberration corrected microscopy. In each of these applications, we extrapolate changes in interfacial atomic and valence electronic structure on the basis of the oxide defect chemistry induced by irradiation. Following experimental results, first-principles based theoretical and energetic predictions are then used to investigate the resolved interfacial chemistry and structure, including mechanical [15] properties. These results provide a critical insight into the properties of these interfaces, their role on radiation damage evolution, and how composite materials can be designed to be more radiation tolerant. 
References:

[1] P.C. Tiemeijer, Phys. Conf. Ser. 161 (1999) 191.

[2] M. Nastasi et al., Phil. Mag. 91 (2011) 553.

[3] Odette et al., Materials Research. 38 (2008) 471.

[4] A. Misra et al., JOM (2007) 62.

[5] B. Rafferty et al., Journal Elect. Micro. 49(4) (2000) 517.

[6] A. Gloter et al., Ultramicroscopy 96 (2003) 385.

[7] P.E. Batson et al., Ultramicroscopy 41 (1992) 137.

[8] R. Buczko et al., Phys. Rev. Lett. 85 (2000) 2168.

[9] C. Hebert, Micron 38 (2007) 12.

[10] K. Jorissen, J.J. Rehr, J. Verbeeck, Phys. Rev. B 81 (2010) 155108.

[11] B. W. Reed and M. Sarikaya, Phys. Rev. B 64 (2001) 195404.

[12] B. W. Reed et al., Phys. Rev. B 60 (1999) 5641.

[13] J. A. Aguiar et al., Ultramicroscopy 124 (2013) 130.

[14] M.P. Prange et al., Phys. Rev. Lett. 109 (2012) 246101.

[15] P. Hosemann et al., J. Nucl. Mater. 12 (2010) 200.

[16] This work was supported by Center for Materials at Irradiation and Mechanical Extremes (CMIME), an Energy Frontier Research Center funded by the U.S. Department of Energy, Office of Science, Office of Basic Energy Sciences under Award Number 2008LANL1026. We would like to specially acknowledge Engang $\mathrm{Fu}$ and Yongqiang Wang at the Ion Beam Materials Laboratory for performing our ion irradiations. We also strongly acknowledge the SHaRE user facility at Oak Ridge National Laboratory where park of the TEM work is performed. We would also like to acknowledge Rob and Pat Dickerson at Los Alamos National Laboratory for successfully preparing our TEM samples. 